\title{
The s-process in globular cluster M22: hints for higher-mass polluters
}

\section{Ian U. Roederer*i}

Carnegie Observatories, 813 Santa Barbara Street, Pasadena, CA 91101, USA

E-mail: iurdobs.carnegiescience.com

\begin{abstract}
Every cool globular cluster star that has been studied in sufficient detail reveals the presence of elements heavier than the iron-group. Surely that must be an important clue to understanding how and where these neutron-capture elements are synthesized. The more complex globular clusters like M22 provide a new kind of opportunity to study nucleosynthesis patterns produced by the slow neutron-capture reaction, or s-process. This provides a new constraint on the duration of star formation in M22. This proceeding is a transcript, edited only to capture the essence of images shown on the slides, of the talk I presented on the subject of s-process material in M22.
\end{abstract}

XII International Symposium on Nuclei in the Cosmos

August 5-12, 2012

Cairns, Australia

${ }^{*}$ Speaker.
${ }^{\dagger}$ I.U.R. is supported by the Carnegie Institution for Science through a Carnegie Fellowship. 


\section{Heavy Elements in Globular Clusters}

Observations of the high-redshift Universe tell us that galaxies destined to grow like the Milky Way formed many of their stars in the first one to three billion years after the Big Bang. During that time, vigorous heavy metal enrichment had already begun. A fair amount of this enrichment apparently occurred in regions that would evolve into globular clusters, since all late-type globular cluster stars studied contain—not just detectable, but significant amounts of-elements from iron to thorium and likely uranium. That is quite remarkable, I think.

Here I would like to focus on the stars responsible for creating these heavy elements. Over the last 20 years or so, observations have demonstrated that the bulk of the heavy elements in most metal-poor globular clusters were produced by r-process nucleosynthesis. ${ }^{1}$ This implies a fairly rapid formation timescale. There are a few metal-poor globular clusters whose stars show evidence of enrichment by both the r-process and s-process. ${ }^{2}$ These clusters are potentially quite interesting because the timescales of heavy element enrichment and cluster formation are intimately linked. If you think you understand one, you constrain the other.

Nearly all globular clusters show evidence of multiple stellar populations by intra-cluster starto-star variations in their light element abundances, like carbon, nitrogen, oxygen, and sodium, for example, that cannot be due entirely to nucleosynthesis within these stars. Star-to-star variations among the heavier alpha, iron-group, and neutron-capture elements within a given cluster are rare. I would like to share with you some recent results that suggest an extended yet relatively rapid formation timescale for one globular cluster, M22, that shows variations in all of these element groups. ${ }^{3}$

\section{M22: A Unique Opportunity To Study s-process Nucleosynthesis}

M22 (NGC 6656) is a massive $\left(M \approx 4 \times 10^{5} M_{\odot}\right)$, metal-poor $([\mathrm{Fe} / \mathrm{H}] \approx-1.75)$ globular cluster that contains several different stellar populations. This was discussed in great detail in Reference [3]. All of these stellar populations were enriched by the r-process, and some contain an additional dose of heavy elements produced by the s-process. Now of course the elements from these two processes are completely mixed in the stars as we observe them. Rather than just trying to quantify the relative proportions of $\mathrm{r}$ - and s-process material, we are presented with a rare opportunity because these two groups are contained within a single cluster.

Last year we made some new observations of three stars in each group, selected based on the prior knowledge about their basic heavy element abundances from Reference [3]. We used the MIKE spectrograph on the Magellan-Clay Telescope at Las Campanas Observatory in Chile. We obtained high signal-to-noise ( $\mathrm{S} / \mathrm{N} \sim 40$ at $3950 \AA$, gradually increasing to $\mathrm{S} / \mathrm{N} \sim 300$ at $6750 \AA$ ) at high spectral resolution $(\lambda / \Delta \lambda \sim 35,000-41,000)$ across the full optical wavelength range for each of these stars. This allowed us to derive abundances for 44 species of 40 elements-almost half of the periodic table-including 24 elements heavier than the iron group.

\footnotetext{
${ }^{1}$ See, e.g., Reference [1].

${ }^{2}$ These clusters include M22, NGC 1851, and $\omega$ Centauri.

${ }^{3}$ These results have already been published in Reference [2]. Here, I present a summary of those results and provide some context for interpretation.
} 


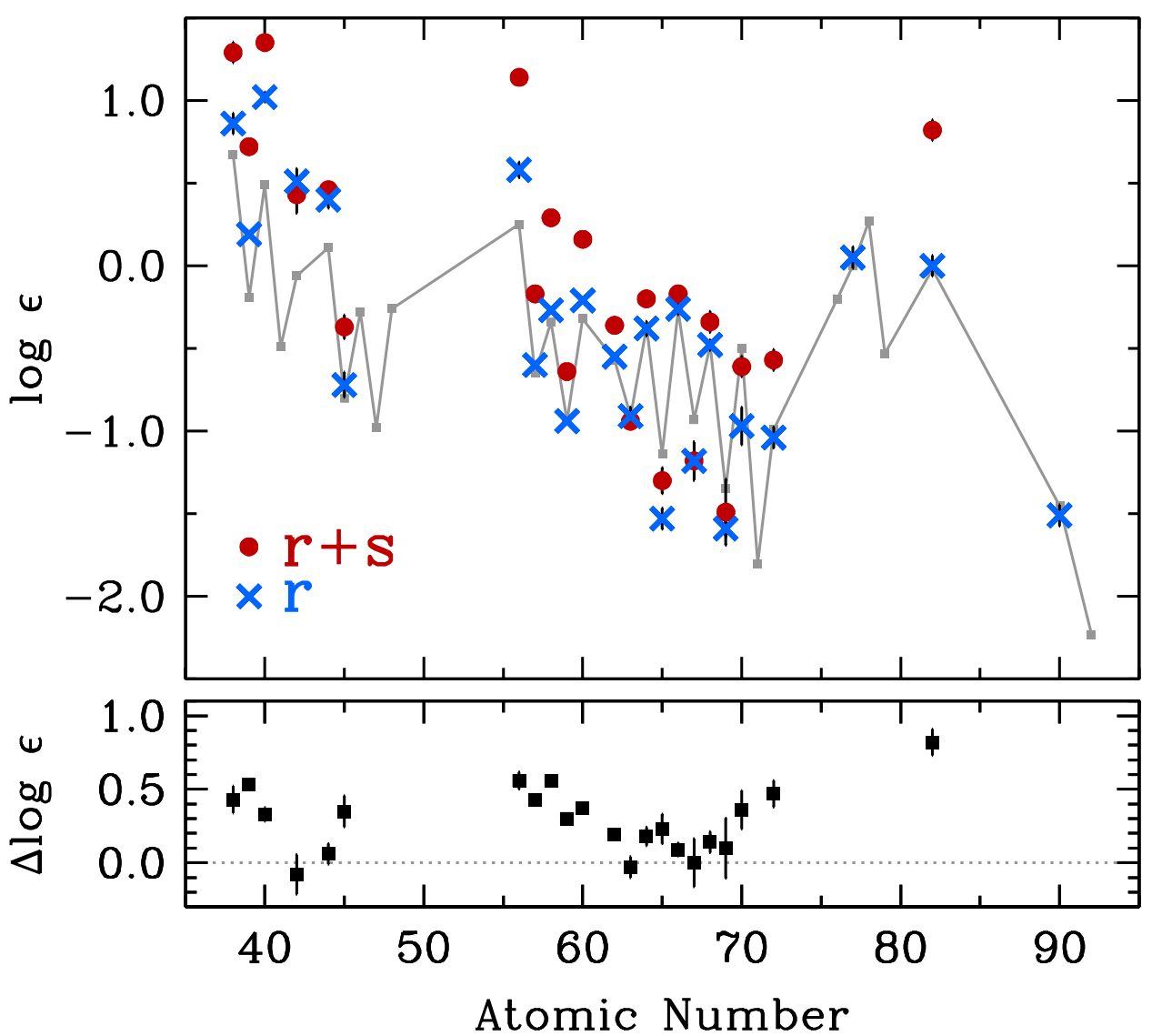

Figure 1: The top panel shows the mean logarithmic abundances for the three r-only stars (blue crosses) and the three $\mathrm{r}+\mathrm{s}$ stars (red circles) as a function of atomic number. The gray symbols mark the abundances in the r-process rich standard star BD+17 3248 (References [4]-[7]), normalized to the europium abundance $(Z=63)$. The bottom panel shows the differences in these two abundance patterns, in the sense of $r+s$ minus r-only. This figure is a reprint of Figure 4 of Reference [2].

What does this do for us? We start by deriving the abundance pattern from the first group of stars, which is shown in the top panel of Figure 1. And you get some pattern, which is shown by the blue crosses. It turns out that this pattern is consistent with what you might expect if only the r-process contributed to the heavy element inventory in these stars. I will refer to this pattern as the "r-process only" pattern. Then we derive the pattern from the other group of stars, shown by the red dots. I will refer to this pattern as the " $\mathrm{r}+\mathrm{s}$ " pattern. We subtract one from the other and are left with the intrinsic s-process "residual".

This is the unique opportunity. You cannot do this for stars in the field or in most globular clusters. The novel feature here is that we know exactly what the underlying r-process pattern is. We do not have to assume anything about the Solar system r-process distribution, or the r-process distribution found in very metal-poor r-process rich standard stars, or anything else. In fact, it does not even matter that it is an r-process pattern! We just need to know how much to subtract off to reveal the heavy element pattern of the new material that was added to the " $r+s$ " group of stars. 
The bottom panel of Figure 1 shows the abundance pattern of the s-process residual. You can see that it is more strongly concentrated toward the three s-process peaks—strontium, barium, and lead-and less so for elements whose Solar abundance is typically attributed mainly to r-process nucleosynthesis, like many of the rare earth elements. This, of course, suggests that some kind of s-process was responsible.

We can construct the ratios of key elements in the s-process material, $[\mathrm{Pb} / \mathrm{La}]$ or $[\mathrm{hs} / \mathrm{ls}],{ }^{4}$ for example. ${ }^{5}$ We then compare these ratios with model predictions for asymptotic giant branch (AGB) stars of various masses at low metallicity. These predictions are made by many investigators, including S. Bisterzo, R. Gallino, S. Cristallo, S. Goriely, A. Karakas, M. Lugaro, and others. When compared to the theoretical yields, the M22 ratios point to a common theme: AGB stars with masses below $3 M_{\odot}$ cannot reproduce the observed values unless the standard ${ }^{13} \mathrm{C}$ pocket efficiency is reduced by factors of 30 to 150 . In effect, this says that the ${ }^{13} \mathrm{C}(\alpha, n){ }^{16} \mathrm{O}$ reaction is not really doing much.

This does not need to be surprising. In M22, lead is enhanced relative to the lighter elements and iron, but it is not nearly as enhanced as observed in metal-poor stars extrinsically enriched in s-process elements by a low-mass AGB binary companion. AGB stars with higher masses may activate the ${ }^{22} \mathrm{Ne}(\alpha, n){ }^{25} \mathrm{Mg}$ reaction but not the ${ }^{13} \mathrm{C}(\alpha, n){ }^{16} \mathrm{O}$ reaction. The result is that the $[\mathrm{Pb} / \mathrm{La}]$ ratios are lower. Unfortunately these predictions are quite limited in terms of the grid of AGB mass, metallicity, and number of elements. But a few key ratios from the higher-mass AGB predictions suggest this is at least a possibility worth testing in greater detail, and there are many more elements available to compare with in our observed distribution.

\section{Implications for the Formation Timescale of M22}

Let us consider one implication of this. Models of stellar evolution can be used to predict the lifetimes of stars of different initial masses. Of course there are some uncertainties in the input physics. For a $20 M_{\odot}$ star, the lifetime is of order $10 \mathrm{Myr}$. For a $10 M_{\odot}$ star, the lifetime is of order $30 \mathrm{Myr}$. For a $5 M_{\odot}$ star, the lifetime is of order 80 to $100 \mathrm{Myr}$. For a $3 M_{\odot}$ star, the lifetime is of order 250 to $350 \mathrm{Myr}$. If you can link the chemistry of the present-day stars with a particular mass range of earlier stars, this naturally sets the timescale for chemical enrichment in the globular cluster. We assume that the gas from which all stars in the cluster formed was pre-enriched with metals from previous generations of stars. This includes the iron-group elements and the r-process material, and it affects all stars in the present-day cluster. As time moves forward, stars with lower and lower masses die and pollute the cluster ISM. Stars more massive than about $8 M_{\odot}$ probably die as supernovae, and their chemical yields seem to be mostly but not entirely lost from the starforming gas in the cluster. Perhaps AGB stars with masses below $8 M_{\odot}$ contribute to the familiar oxygen-sodium anti-correlation seen in M22 and other globular clusters. If we accept that the sprocess pattern in the " $\mathrm{r}+\mathrm{s}$ " group in M22 was not produced by AGB stars with initial masses lower

\footnotetext{
${ }^{4} \mathrm{Here},[\mathrm{hs} / \mathrm{ls}]$ is defined as $[\mathrm{hs} / \mathrm{Fe}]-[1 \mathrm{~s} / \mathrm{Fe}]$, where $[\mathrm{hs} / \mathrm{Fe}]$ is defined as $\frac{1}{3}([\mathrm{La} / \mathrm{Fe}]+[\mathrm{Nd} / \mathrm{Fe}]+[\mathrm{Sm} / \mathrm{Fe}])$ and $[1 \mathrm{~s} / \mathrm{Fe}]$ is defined as $\frac{1}{2}([\mathrm{Y} / \mathrm{Fe}]+[\mathrm{Zr} / \mathrm{Fe}])$.

${ }^{5}$ Other useful indicators, such as the $[\mathrm{Rb} / \mathrm{Zr}]$ ratio, cannot be derived in the $\mathrm{M} 22$ stars because the $\mathrm{Rb}$ I lines are undetected and only yield uninteresting upper limits. Furthermore, the $[\mathrm{Cu} / \mathrm{Fe}]$ and $[\mathrm{Cu} / \mathrm{Zn}]$ ratios may be of interest, but models of s-process nucleosynthesis in AGB stars appropriate to M22 have not yet been published for these elements.
} 
than about $3 M_{\odot}$, this constrains the time over which new stars were forming in M22, something like $300 \mathrm{Myr}$ or less after the first surviving stars in M22 formed.

Interestingly, an independent line of study reaches the same conclusion for M22, as revealed by Reference [8]. Figure 12 of that study reveals a split subgiant branch on the color-magnitude diagram, and the split corresponds to the " $r$-only" and " $r+s$ " groups. That study also characterized the different carbon, nitrogen, and oxygen abundances in stars on each of the two branches. You can explain the split just by the differing stellar compositions of carbon, nitrogen, and oxygen. If you use that to set the abundances in the stellar models, the isochrones naturally fit the split subgiant branch with a negligible age spread with an uncertainty — or maximum duration of star formation—of about $300 \mathrm{Myr}$.

This is quite interesting, I think, because two independent approaches both lead to consistent results that suggest that M22-as strange as its chemistry is, and there are many other questions left unanswered ${ }^{6}$ - formed in no more than a few hundred million years. M22 is not quite unique among Galactic globular clusters, but those like it are rare. In a few very localized regions of the early stellar halo, more massive AGB stars may have played an important role in the chemical evolution of the heavy elements.

\section{Questions and Discussion}

W. Aoki: Do you find any scatter in the $[\mathrm{Eu} / \mathrm{Fe}]$ ratios in the "r-only" stars?

I.U.R.: No. There is a small but measurable distribution of $[\mathrm{Fe} / \mathrm{H}]$ in $\mathrm{M} 22$, but the $[\mathrm{Eu} / \mathrm{Fe}]$ ratios are constant.

A. Alves-Brito: Can you comment on the relationship between the light elements and heavy elements that you find for the two different groups in M22?

I.U.R.: The oxygen-sodium anti-correlation is present in both stellar groups in M22, although the distributions may be slightly different. Your work, as well as work by Anna Marino and Valentina D'Orazi, has shown that the light elements from carbon through aluminium are not constant among the two groups. I think we could really make progress by trying to find a set of AGB models that can simultaneously reproduce the changes in the light elements and the s-process elements in M22. This could really help pin down the mass range of stars that contributed to the chemical inventory. Such a set of models has not been tested as far as I know.

J. Danzinger: Most of the discussion on s-processes in stars has focused on AGB stars. But the most obvious sources of s-process material are barium stars which McClure showed were always low mass binary systems.

I.U.R.: That is a perfectly reasonable explanation for s-process enrichment in field stars where the binary fraction is high. There the s-process material is attributed to a companion that passed

\footnotetext{
${ }^{6}$ For example, there is no correlation between the s-process material and the products of hot bottom burning in M22, despite our understanding that more massive AGB stars may be responsible for producing the oxygen-sodium anti-correlation. The lack of large amounts of s-process material in other clusters that show evidence of the oxygensodium anti-correlation is also puzzling.
} 
through the AGB phase of evolution. However, the binary fractions in globular clusters are just a few percent or less. Even if this fraction was higher initially, it could not account for other observed properties in M22, such as the positive correlation between s-process enrichment and metallicity.

\section{References}

[1] Roederer, I.U. Primordial r-process Dispersion in Metal-Poor Globular Clusters, Astrophys. J. Letters 732, L17 (2011)

[2] Roederer, I.U., Marino, A.F., Sneden, C. Characterizing the Heavy Elements in Globular Cluster M22 and an Empirical s-process Abundance Distribution Derived from the Two Stellar Groups, Astrophys. J. 742, 37 (2011)

[3] Marino, A.F., Sneden, C., Kraft, R.P., Wallerstein, G., Norris, J.E., Da Costa, G., Milone, A.P., Ivans, I.I., Gonzalez, G., Fulbright, J.P., Hilker, M., Piotto, G., Zoccali, M., Stetson, P.B. The two metallicity groups of the globular cluster M22: a chemical perspective, Astron. \& Astrophys. 532, A8 (2011)

[4] Cowan, J.J., Sneden, C., Burles, S., Ivans, I.I., Beers, T.C., Truran, J.W., Lawler, J.E., Primas, F., Fuller, G.M., Pfeiffer, B., Kratz, K.-L. The Chemical Composition and Age of the Metal-Poor Halo Star $B D+17^{\circ} 3248$, Astrophys. J. 572, 861 (2002)

[5] Cowan, J.J., Sneden, C., Beers, T.C., Lawler, J.E., Simmerer, J., Truran, J.W., Primas, F., Collier, J., Burles, S. Hubble Space Telescope Observations of Heavy Elements in Metal-Poor Galactic Halo Stars, Astrophys. J. 627, 238 (2005)

[6] Sneden, C., Lawler, J.E., Cowan, J.J., Ivans, I.I., Den Hartog, E.A. New Rare Earth Element Abundance Distributions for the Sun and Five r-process-rich Very Metal-Poor Stars, Astrophys. J. 182, 80 (2009)

[7] Roederer, I.U., Sneden, C., Lawler, J.E., Cowan, J.J. New Abundance Determinations of Cadmium, Lutetium, and Osmium in the r-process Enriched Star BD +17 3248, Astrophys. J. Letters 714, L123 (2010)

[8] Marino, A.F., Milone, A.P., Sneden, C., Bergemann, M., Kraft, R.P., Wallerstein, G., Cassisi, S., Aparicio, A., Asplund, M., Bedin, R.L., Hilker, M., Lind, K., Momany, Y., Piotto, G., Roederer, I.U., Stetson, P.B., Zoccali, M. The double sub-giant branch of NGC 6656 (M22): a chemical characterization, Astron. \& Astrophys. 541, A15 (2012) 